\title{
Autoritarismo Competitivo y Elecciones en Ecuador
}

\author{
Arturo Moscoso Moreno, Melina Villavicencio Mancero
}

Recepción: 16 de septiembre Aprobación: 11 de noviembre

\section{Resumen}

Este trabajo analiza, a la luz de la votación y el número de asambleístas provinciales obtenidos por Alianza PAIS en las elecciones de 2009, 2013 y 2017, la estructuración del sistema electoral ecuatoriano a partir de la aprobación de la Constitución de 2008, su proporcionalidad y las fórmulas de asignación de escaños utilizadas. Se estudia el campo de juego electoral en el cual han competido la oposición y el oficialismo, determinando su desnivel a favor de los segundos, ajustándose a una de las características esenciales de lo que Levitsky y Way han denominado autoritarismos competitivos.

\section{Palabras clave}

Reforma electoral, autoritarismo competitivo, democracia, elecciones, fórmulas de asignación de escaños, Alianza PAIS

\section{Abstract}

This paper analyzes, in light of the voting and the number of legislators obtained by Alianza PAIS in the elections of 2009, 2013 and 2017, the structure of the Ecuadorian electoral system since the approval of the 2008 Constitution, its proportionality and the seat allocation formulas used. It is also studied the electoral playing field in which the opposition and the ruling party have competed, determining the unevenness in favor of the latter, which adjusts to one of the essential characteristics of the competitive authoritarianisms in the terms of Levitsky and Way.

\section{Key Words}

Electoral reform, competitive authoritarianism, democracy, elections, seat allocation formulas, Alianza PAIS 


\section{Introducción}

ntre los académicos y estudiosos de la democracia existe una preocupación creciente sobre lo que se ha denominado la "recesión democrática"; una disminución gradual de los atributos de los regímenes democráticos, que ya no se quiebran a través de golpes de estado, sino a manos de líderes democráticamente electos que subvierten lenta y paulatinamente el proceso mediante el cual fueron electos, manteniendo una fachada democrática (Diamond 2015; Foa y Munck 2016; Levistky y Ziblatt 2018; Lührmann y Lindberg 2019). Según Levitsky y Ziblatt (2018), el retroceso democrático empieza en las mismas urnas de votación.

Esta progresiva disminución de los requisitos democráticos conduce a procesos de autocratización que si bien no arriban a autoritarismos plenos, dan como resultado regímenes o democracias disminuidas, imperfectas o de baja calidad que, dependiendo de los atributos de la democracia que sus autores consideran afectados, pueden ser definidas de distintas formas. Así, se encuentran conceptos como "regímenes semidemocráticos" (Mainwaring, et al. 2002), “democracias delegativas" (O’Donnel 1997), "democracias iliberales" (Zakaria 1998), "regímenes híbridos" (Diamond 2009), "democracias y autoritarismos electorales" de Schedler (2006) o "autoritarismos competitivos" (Levitsky y Way 2009), concepto que será el que se utilizará en este trabajo porque se considera que es el que mejor explica el proceso de autocratización del régimen de Rafael Correa (Lührmann y Lindberg 2019; Moscoso 2013; Pachano y Garcia 2015).

Los autoritarismos competitivos son regímenes en que existen instituciones democráticas formales y son consideradas ampliamente como los medios para obtener y ejercer la autoridad política, pero en los que funcionarios electos violan estas reglas con frecuencia y se aprovechan de su cargo para obtener ventajas sobre sus oponentes. Existe competencia electoral, pero no es justa (Levitsky y Way 2010: 17).

Para Levitsky y Way, lo que distingue al autoritarismo competitivo de la democracia es el hecho de que el titular del Estado viola al menos uno de los tres atributos que definen a la democracia: (1) elecciones libres, (2) una 
amplia protección de las libertades civiles, y (3) un campo de juego nivelado para todos sus actores. En las democracias, señalan, las elecciones son libres cuando no hay fraude e intimidación a los votantes; y son justas cuando los partidos de oposición pueden hacer campaña en un campo de juego relativamente nivelado, en donde no sean sujetos de represión o acoso o se les niegue sistemáticamente acceso a los medios o a otros recursos críticos (2010).

En los autoritarismos competitivos, si bien las elecciones son competitivas, rara vez un contendiente es excluido y la oposición puede hacer campaña libremente, son frecuentemente injustas y casi nunca libres. Entre las características que destacan Levitsky y Way para que esto ocurra, están un proceso de conteo de votos injusto en una cancha de juego desnivelada (2010: 366).

El 19 de febrero de 2017 se llevaron a cabo las últimas elecciones generales en Ecuador hasta la fecha, en las cuales se eligieron, entre otros funcionarios, a 116 legisladores o asambleístas provinciales que pasaron a conformar, junto con 15 asambleístas de circunscripción nacional y 6 por circunscripciones del extranjero, el pleno del tercer período legislativo de la Asamblea Nacional desde su instauración en 2008².

Los resultados finales fueron proclamados por el Consejo Nacional Electoral (CNE) el 26 de febrero de 2017, en los cuales, por segunda vez consecutiva, Alianza PAIS (AP) obtuvo la mayoría absoluta -aunque perdió la mayoría calificada de los dos tercios de escaños que mantenía desde las elecciones de 2013-, haciéndose con 67 escaños provinciales, 7 nacionales y 4

\footnotetext{
1.-De acuerdo al Art. 150 de la Ley Orgánica Electoral y de Organizaciones Políticas de la República del Ecuador, Código de la Democracia (LOEOP), se eligen 15 asambleístas en circunscripción nacional; 2 asambleístas por cada provincia o distrito metropolitano, y uno más por cada doscientos mil habitantes o fracción que supere los 150.000, de acuerdo al último censo - 116 en el caso de las últimas elecciones-; 6 asambleístas por las circunscripciones especiales del exterior distribuidos así: 2 por Europa, Oceanía y Asia, 2 por Canadá y Estados Unidos y 2 por Latinoamérica, el Caribe y África. Las circunscripciones electorales que eligen entre 8 y 12 representantes se subdividen a su vez en 2 circunscripciones, aquellas que pasan de 13 y hasta 18 se subdividen en 3 y las que pasan de 18 se subdividen en 4 circunscripciones. Al momento, las provincias de Pichincha y Guayas están divididas en 4 distritos cada una y la de Manabí en dos.
} 
del extranjero, para un total de 74 de los 137 en disputa ${ }^{2}$. Con el 39,07\% de los votos, AP obtuvo casi el $50 \%$ de los escaños de asambleístas provinciales en juego; esto es una diferencia de más de 11 puntos porcentuales entre la votación que obtuvo y los escaños que le fueron asignados mediante el conteo de votos, evidenciando una marcada desproporcionalidad del sistema electoral ${ }^{3}$.

El propósito de este trabajo es analizar, a la luz de la votación y el número de asambleístas provinciales obtenidos por Alianza PAIS en las elecciones de 2009, 2013 y 2017, si es que el campo de juego en el cual han competido la oposición y el oficialismo estuvo desnivelado a fin de favorecer a los segundos, configurando las características del autoritarismo competitivo descritas por Levitsky y Way, para lo cual se analizará la estructuración del sistema electoral ecuatoriano, a partir de la aprobación de Constitución de 2008, su proporcionalidad y las fórmulas de asignación de escaños utilizadas ${ }^{4}$.

\section{La cancha inclinada}

Para Levitsky y Way, la evidencia que demuestre la parcialización sistemática de las autoridades electorales en favor del oficialismo y un acceso altamente desigual a los medios y a los recursos, constituyen un campo de juego desnivelado. A su vez, para que exista un campo de juego desnivelado, basta con la existencia de al menos una de las siguientes 3 características:

1. El amplio abuso de las instituciones estatales con fines partidistas;

2.- En las elecciones generales de 2013 , AP obtuvo 100 escaños de un total de 137 , es decir el $73 \%$ de la Asamblea con el 52,30\% de los votos (CNE, 2016)

3.-El Índice de Gallagher (IG), o índice de mínimos cuadrados, mide la desproporción en los resultados electorales, calculando la diferencia entre el porcentaje de votos recibidos y el porcentaje de escaños que ha obtenido un partido en la legislatura resultante. De acuerdo con este índice, un valor mayor a diez puntos entre la proporción de votos recibidos y la proporción de escaños adjudicados hace presumir que el sistema electoral tiene una clara tendencia mayoritaria. En Ecuador, este promedio fue superior a 24,9, lo que lleva a concluir que nuestro sistema electoral, pese a utilizar fórmulas proporcionales, es un sistema en realidad mayoritario (Gallagher, 1991; Ortiz, 2016: 24).

4.- Ecuador ha experimentado cambios constantes de las reglas electorales desde la aprobación de la Constitución de 1978 que permiten identificar al menos cuatro sistemas electorales distintos, con cambios en diversos elementos del sistema electoral como la estructura del voto, el principio de representación, el tamaño del legislativo, la fórmula electoral, el tamaño de los distritos electorales y la barrera electoral (Freidenberg, 2011: 17). 
2. El oficialismo es sistemáticamente favorecido a expensas de la oposición; y,

3. La posibilidad de la oposición para organizarse y competir en elecciones está seriamente limitada (2010: 366-368).

Dentro de los aspectos más importantes a considerar en un campo de juego inclinado están el acceso a los recursos, a los medios y a la ley. El acceso a ésta última se ve severamente limitado cuando se nombra árbitros sesgados, que regulan sistemáticamente a favor del oficialismo, violando incluso los procedimientos democráticos (Levitsky y Way 2010: 12).

Mediante consulta popular, llevada a cabo en abril de 2007, en el primer año del gobierno de Rafael Correa y Alianza PAIS, se aprobó, por una mayoría del $81,72 \%$ de los votantes, la creación de una nueva Constitución para Ecuador, para lo cual se convocó a elecciones en septiembre del mismo año para elegir a quienes conformarían la Asamblea Constituyente que la elaboraría $^{5}$. En septiembre del mismo año se eligieron 130 asambleístas, adjudicándose a Alianza PAIS más del 70\% de los escaños.

5.- Rafael Correa se posesionó como presidente el 15 de enero de 2007 y, como había ofrecido en su campaña, firmó el Decreto 002 mediante el cual solicitó al Tribunal Supremo Electoral (TSE) que llame de manera inmediata a consulta popular para que se decida convocar a una Asamblea Constituyente "de plenos poderes" para redactar una nueva Constitución. La convocatoria la amparó en lo manifestado en el Art. 283 y en el numeral 2 del Art. 104 de la Constitución de 1998, pero eliminó la frase "distintas de las previstas en el número anterior", lo que constituyó una violación flagrante de los artículos previstos en la carta política, primero por no ajustarse a lo estipulado en el Art. 283 del mismo cuerpo legal que hablaba únicamente de reformas y no de asambleas, y luego, por la ilegal mutilación del Art. 104 que desvirtuaba totalmente su sentido. Recibida la convocatoria, el TSE lo remitió al Congreso, donde fue rechazado por la mayoría legislativa que estaba conformada por legisladores del Partido Sociedad Patriótica (PSP), Partido Social Cristiano (PSC), Partido Renovador Institucional Alvaro Noboa (PRIAN) y la Unión Demócrata Cristiana (UDC), que pretendía reformar la Constitución por vía legislativa. Correa envió un nuevo llamado al TSE con la amenaza de nombrar un Tribunal Electoral Ad Hoc si no se convocaba a consulta, lo que este organismo hizo sin remitirlo al Congreso, anunciando, con fecha 13 de febrero de 2007 que la consulta se llevaría a cabo con fecha de 15 de abril de ese año.

Ante esto, una mayoría legislativa de 57 diputados, con fecha 6 de marzo, votó a favor de la destitución del presidente del TSE, la titularización de su suplente y el llamado a juicio político para los cuatro vocales que aprobaron la convocatoria a consulta popular. El 7 de marzo de 2007, el TSE con el voto de 4 de sus 7 vocales destituyeron a los 57 diputados con el argumento de que el TSE, al haberse iniciado un proceso electoral, podía destituir a cualquier funcionario que intentara oponerse al mismo, de acuerdo con lo que expresaba el literal e) del Art. 155 de la Ley de Elecciones vigente a la época.

Más de un mes después, el Congreso volvió a sesionar con los diputados suplentes de los destituidos (conocidos como los "diputados de los manteles" por haber huido de la prensa, ocultos bajo los manteles de las mesas del restaurante de una hostería en las afueras de Quito en la que se habían reunido con representantes del gobierno), que en su mayoría habían sido cooptados por el oficialismo, con lo que se aseguró la mayoría en el Congreso. 
Así, el 28 de septiembre de 2008 se aprobó vía referéndum, por una mayoría de más del $60 \%$ de la votación, la Constitución actualmente vigente del Ecuador, que rompió con la clásica división de poderes, creando dos nuevas funciones del Estado: la Función de Transparencia y Control Social y la Función Electoral (Constitución de la República, 2008, Art. 204, Art. 217).

La Función de Transparencia y Control Social está integrada por la Contraloría General del Estado, la Defensoría del Pueblo, las Superintendencias y el Consejo de Participación Ciudadana y Control Social (CPCCS), que es su órgano ejecutivo y la entidad que nombra entre otros funcionarios, a los miembros del Consejo Nacional Electoral (CNE) y a los del Tribunal Contencioso Electoral (TCE), arrebatándole el poder nominador al legislativo. A su vez, los consejeros miembros del CPCCS son elegidos mediante un proceso de selección organizado por el CNE.

A fin de posibilitar todos los cambios institucionales que trajo la Constitución de 2008, se implementó un "Régimen de Transición", cuyas primeras acciones fueron las de cesar a todos los legisladores y conformar una Comisión Legislativa y de Fiscalización, denominada coloquialmente "Congresillo", a fin de cumplir las tareas de la Asamblea Nacional hasta que se eligieran nuevos legisladores. Esta entidad quedó conformada con una mayoría oficialista (Constitución de la República, 2008, Régimen de Transición) ${ }^{6}$.

En relación con la Función Electoral, el régimen de transición determinó que fuera la Asamblea Constituyente la que designe a un CNE transitorio, que luego sería reemplazado por el que se eligiere en los procesos que llevare acabo el CPCCS. Esta tarea la llevó a cabo el 24 de octubre de 2008, designando, sin concurso, a Omar Simon, Fausto Camacho, Manuela Cobacango, Marcia Caicedo y Carlos Cortez con los votos de la mayoría oficialista de Alianza PAIS; todos con estrechas vinculaciones al oficialismo ${ }^{7}$ (El Comercio 2010).

6.- La Comisión Legislativa y de Fiscalización estuvo integrada por 76 miembros, elegidos por el pleno de la Asamblea Constituyente, asignando la mayor cantidad de escaños a los partidos más votados en la elección de Asambleístas constituyentes de 2007, lo que dio a Alianza PAIS una mayoría absoluta de 46 miembros. 
De igual forma, la disposición transitoria segunda de la Constitución de 2008 determinó que el "Congresillo" fuera el que designe, con base en un concurso público de oposición y méritos, a los primeros consejeros del CPCCS, quienes permanecerían en sus funciones hasta que se promulgue la ley que regule la organización y funcionamiento de esa entidad (Constitución de la República, 2008, Régimen de Transición).

Efectivamente, en enero de 2009, fueron designados, por concurso llevado a cabo por la comisión especializada de Participación Social del "Congresillo," los miembros del CPCCS transitorio, quienes no cumplieron ninguna función relevante ni nombraron ninguna autoridad pública, y fueron cesados el 9 de septiembre de 2009, luego de la promulgación de la Ley del Consejo de Participación Ciudadana y Control Social, conforme señalaba el régimen de transición de la Constitución de 2008, quedando a cargo una administradora temporal y procediéndose a llamar a concurso para integrar el CPCCS definitivo, que sería manejado por el CNE transitorio, afín al oficialismo.

Se postularon 367 personas, cuyas carpetas fueron analizadas por una "Comisión de Apoyo" integrada por los asesores de los vocales del CNE Francisco Morales, brazo derecho de Simon; Mireya Jiménez; Carlos Novillo, que fuera coordinador nacional de la Federación de Estudiantes Universitarios del Ecuador (FEUE), cercana al Movimiento Popular Democrático (MPD), partido aliado del oficialismo a esa época; Natalia Cantos, que fuera secretaria de Novillo, y Miguel Ángel Condolo, cercano a la FENOCIN, agrupación indígena de izquierda, incondicional al gobierno de Rafael Correa (El Comercio 2010).

\footnotetext{
7.- Ricardo Patiño fue asesor y Subsecretario General de Rafael Correa durante su periodo como Ministro de Economía en 2005; luego fue primer secretario ejecutivo del Movimiento Alianza PAIS y durante el gobierno de Correa ocupó los Ministerios de Economía y Finanzas, del Litoral, de Coordinación de la Política y Gobiernos Autónomos Descentralizados, de Relaciones Exteriores y Movilidad Humana y de Defensa Nacional. La esposa de Simon es sobrina de quien fuera ministro de Seguridad Interna y Externa de Rafael Correa, Gustavo Larrea. Fausto Camacho fue asesor del asambleísta de AP Virgilio Hernández en la Asamblea Constituyente que promulgaría la Constitución de 2008. Manuela Cobacango era muy cercana al asambleísta de Alianza PAIS Pedro de la Cruz. Carlos Cortez y, sobre todo, Marcia Caicedo, mostrarían cierta independencia (El Comercio, 2010).
} 
De esta forma, la misma tendencia de cercanía al oficialismo se repitió en el CPCCS definitivo. Los vocales elegidos por el CNE transitorio fueron Juana Luisa Miranda, cercana a la esposa de Ricardo Patiño, una de las principales piezas del gobierno de Rafael Correa; Patricia Ordeñana, quien fuera asesora de Patiño; Mónica Banegas, que fuera parte del CPCCS transitorio y que mantenía simpatías con el gobierno; Fernando Cedeño, quien fuera secretario de Ricardo Patiño en el Ministerio de Economía; Luis Pachala, David Rosero y Andrea Rivera, estos dos últimos miembros del MPD, que poco tiempo después rompería su alianza con el gobierno, convirtiéndolos en voces críticas dentro del organismo, pero en minoría ${ }^{8}$ (El Comercio 2010).

Este CPCSS, claramente cercano al gobierno de AP, se encargó a su vez de la selección de los miembros del CNE definitivo, siendo elegidos en noviembre de 2011 Juan Pablo Pozo, Roxana Silva, Domingo Paredes, Nubia Villacís y Paúl Salazar. Todos ex funcionarios del gobierno de Alianza PAIS o cercanos colaboradores. No fue nombrado ni un solo miembro independiente o peor aún, de la oposición'. Este nuevo CNE organizó los comicios de 2013, pero antes, propondría una reforma clave a ley electoral, de la que se hablará más adelante ${ }^{10}$ (Expreso 2011; El Universo 2012; Sanchez-Sibony 2017).

8.- A partir del rompimiento del MPD con AP, Rosero denunció permanentemente la injerencia gubernamental en las decisiones del CPCCS y en general, en las otras funciones del estado. Rosero luego terminaría detenido y condicionado a presentarse ante las autoridades cada 15 días por las protestas que encabezaría frente a la decisión del gobierno de trasladar los fondos de cesantía del magisterio de Ecuador a la administración del Banco del Instituto Ecuatoriano de Seguridad Social.

9.- Domingo Paredes, hasta ese momento presidente del CNE, fue sorteado en noviembre de 2014 junto con Roxana Silva para ser cesados en sus cargos conforme mandato Constitucional. Fueron reemplazados por Carlos Tayupanta Noroña, asesor de Domingo Paredes en el mismo Consejo y Gloria Toapanta, quien renunciaría a los 6 días de posesionada, alegando "motivos personales". Fue reemplazada por Ana Marcela Paredes, quien fuera Directora de Promoción Electoral en el Instituto Nacional de la Democracia, organismo dependiente del CNE.

Juan Pablo Pozo, a esa fecha presidente del CNE, fue asesor de Fernando Cordero en la Asamblea Nacional, quien fuera presidente de la misma y figura fuerte de Alianza País. A la salida de Domingo Paredes, Pozo ocupó la presidencia del CNE al reconsiderarse la sesión en la que Paúl Salazar fuera electo antes con los votos de Mauricio Tayupanta y Gloria Toapanta. En la sesión de reconsideración, Ana Marcela Paredes, reemplazó de Toapanta, votó por Pozo. La sesión de reconsideración fue propuesta por el mismo Salazar, como presidente del CNE, el que convocó al pleno y la propuso.

El 2 de abril de 2017, en la inauguración de la segunda vuelta electoral de las elecciones generales de ese año, Pozo declaraba: "Entre los mejores recuerdos y acciones que me llevaré cuando concluya la presidencia del CNE es haber coincidido históricamente con el presidente Rafael Correa. Siempre hemos establecido las articulaciones y coordinaciones que conlleva la Función Electoral, pero jamás ha tenido condicionamientos ni presiones". (El Universo, 2017). 


\section{Las elecciones injustas}

El sistema electoral, desde una definición estricta, es el conjunto de reglas que estipula el procedimiento por el que los electores votan y los votos se convierten en escaños en el caso de los miembros del poder legislativo, o en cargos de gobierno en caso de la elección del poder ejecutivo. Son, entonces, las reglas de juego de las elecciones. Este concepto incluye 4 componentes básicos:

1. Las formas de candidatura;

2. El procedimiento de votación;

3. La circunscripción electoral; y,

4. Las formas de conversión de votos en escaños (Ortiz 2016: 8-10).

Las formas de candidatura son dos: personal y por lista. La primera hace referencia a la candidatura de una sola persona, y la segunda a varias, teniendo tres variantes: cerrada y bloqueada, en la que el elector tiene un solo voto para elegir a toda la lista; cerrada y no bloqueada, en la que el elector tiene un voto para la lista y un voto preferente para el candidato al que quisiera que se le adjudique el primer escaño; $y$, abierta, en la que el elector tiene tantos votos como escaños. En Ecuador, en la actualidad la forma de lista determinada para asambleístas provinciales es abierta (LOEOP 2009, Art. 120).

El procedimiento de votación tiene relación directa con la forma de la lista y hace referencia a la manera en que el elector vota, si por un candidato (voto personalizado), o por una lista (voto programático). En Ecuador, al existir listas abiertas, el voto es personalizado (Ortiz 2016: 12).

La circunscripción electoral es el ámbito territorial en el que se lleva a cabo una elección y consiste en el número de jurisdicciones en las que se divide un territorio para efectos electorales, como nacional, provincial, distrital, etc. El tamaño de la circunscripción es el número de escaños a repartir a su interior y pueden estar en disputa uno, dos o más. Si se reparte

10.- Cabe señalar que el CPCCS nombra también al Procurador General del Estado, Superintendentes, Defensor del Pueblo, Defensor Público, Fiscal General del Estado, Contralor General del Estado y a los miembros del Consejo Nacional de la Judicatura (Constitución de la República del Ecuador, 2008), cargos que fueron entregados en su totalidad a simpatizantes y/o colaboradores del partido oficialista. 
un sólo escaño en una circunscripción, ésta es uninominal, si se reparte más de un escaño, es plurinominal.

Las circunscripciones en que se adjudican de 2 a 5 escaños son consideradas pequeñas, en las que se adjudican de 6 a 9 escaños son consideradas medianas y en las que se adjudican más de 10 escaños son consideradas grandes (Nohlen 2004: 57).

Para Ortiz, junto con la fórmula de conversión de votos a escaños, que se verá más adelante, el tamaño de la circunscripción es el "elemento más relevante de un sistema electoral y el que tiene mayor influencia en la proporcionalidad entre votos y escaños"; es decir, la combinación de ambos elementos, la fórmula de conversión y el tamaño de la circunscripción hacen al sistema electoral más o menos proporcional (2016: 12).

El punto es que, según el principio democrático, cada voto debe tener el mismo valor o peso, lo que se logra cuando cada escaño representa la misma cantidad de electores. Sin embargo, a través del tamaño de la circunscripción electoral, se puede alterar ese principio, lo que a veces genera desviaciones, algunas aceptables, otras intolerables, porque violan flagrantemente este principio, quitándole al voto casi todo su valor. Nohlen establece una regla: "Cuanto más pequeña es la circunscripción electoral, menor es el efecto proporcional del sistema electoral; esto significa que disminuyen las posibilidades electorales de los partidos pequeños" (2004: 51). Así, cuando se reduce el tamaño de las circunscripciones electorales, se aumenta la desproporción entre votos y escaños.

Adicionalmente, dentro de los sistemas electorales hay dos reglas básicas de decisión: la mayoritaria y la proporcional. Con la primera, el partido que obtiene el mayor número de votos gana todos o la mayoría de los escaños. En la segunda, los escaños se van repartiendo de acuerdo con el porcentaje de votos que obtiene cada partido. Estas reglas responden a dos principios de representación, el de mayoría y el proporcional.

En el sistema de mayoría, un partido que no haya alcanzado la mayoría absoluta de votos (la mitad más uno) podría igual obtener la mayoría de escaños. Por ejemplo, un partido que gana con el $35 \%$ de los votos podría obtener el $60 \%$ 
de los escaños. El sistema proporcional intenta que exista la mayor aproximación entre el porcentaje de votos obtenido por una organización política y el número de escaños que se le adjudique, intentando obtener la mayor representatividad posible y tratando de acercarse al principio democrático de la mejor forma.

Finalmente, a fin de determinar el ganador de un escaño, se debe utilizar una fórmula de cálculo o método que responda a alguno de los dos principios mencionados. Si la regla responde al principio de mayoría, simplemente ganará quien obtenga la mitad más uno de los votos (mayoría absoluta) o quien obtenga la mayor cantidad de votos (mayoría relativa). Si la regla es de decisión proporcional, se deben utilizar ciertas fórmulas matemáticas. Los dos tipos más importantes de fórmulas son las de divisores contínuos y las de cocientes; de las que se hablará en este trabajo son Webster y D'Hondt.

Tanto Webster como D'Hondt son fórmulas de divisores continuos, en las cuales la votación total de la lista - o la de cada candidato sumada en el caso de listas abiertas -se la divide para 1, 3, 5, 7, etc. en el caso del método Webster; y, para 1, 2, 3, 4, 5 y así sucesivamente cuando se utiliza el método D'Hondt, hasta que se asignen todos los escaños. Esos cocientes se ordenan de mayor a menor y se asignan a cada lista los puestos que le correspondan, empezando por los cocientes más altos, hasta completar el número total de representantes a elegirse.

La regla en la adjudicación de escaños por medio de divisores continuos es que mientras mayor es la distancia entre los divisores, más proporcional será el reparto de escaños. Por lo tanto, el método de Webster es más proporcional que el D’Hondt. Así, si se comparan los efectos de la serie de divisores de D'Hondt con los de Webster, se podrá ver que la aplicación de la primera fórmula puede implicar que un partido grande obtenga un escaño más a costa de un partido minoritario (Ortiz 2016: 16; Nohlen 2004: 73) ${ }^{11}$.

11.- "En sistemas proporcionales basados en circunscripciones plurinominales, la decisión obedece a la fórmula proporcional y persigue el objetivo de representar a los partidos en el parlamento según la proporción de votos obtenidos. No obstante, existen en la práctica sistemas electorales en los que la fórmula de decisión no se corresponde con el principio de representación: bajo ciertas condiciones, la fórmula de decisión proporcional puede conducir a la representación mayoritaria (como objetivo de representación). Estas condiciones específicas están dadas en sistemas llamados proporcionales, basados en circunscripciones pequeñas, donde se aplica la fórmula proporcional, pero la representación proporcional de los partidos resulta imperfecta debido a la baja cantidad de escaños en juego. Las circunscripciones pequeñas favorecen fuertemente a los partidos grandes, de manera que en el nivel nacional se presentan diferencias significativas entre la proporción de votos y escaños en perjuicio de los partidos pequeños." (Nohlen, 1995: 48) 
Por su parte, Estrella Aguilar, al comparar los efectos de las fórmulas de Webster y D'Hondt en el sistema electoral legislativo ecuatoriano, utilizando los indicadores d Distorsión, Distorsión Relativa, ISL e Índice de Gallagher, concluye que las distribuciones que genera Webster son los que mejor cumplen el Principio de Proporcionalidad, mientras que las que genera D'Hondt, se alejan considerablemente del mismo (2018: 168 - 169).

\section{El Sistema Electoral para las Elecciones Generales 2009}

En primer lugar, se debe señalar que la Constitución de Ecuador determina en su Art. 116 que la ley debe establecer un sistema electoral conforme a los principios de proporcionalidad, igualdad de voto y equidad, lo que, como se verá más adelante, no se ha cumplido en la práctica (Constitución de la República del Ecuador 2008).

De tal manera, para las elecciones generales que se llevaron a cabo el 26 de abril de 2009, el sistema electoral a utilizarse quedó determinado en el Capítulo II del Régimen de Transición de la Constitución de 2008, estableciéndose, en primer lugar, una forma de lista abierta para la elección de asambleístas nacionales, provinciales, del exterior, concejales y miembros de juntas parroquiales rurales.

En cuanto a la asignación de escaños para asambleístas nacionales, asambleístas provinciales, asambleístas del exterior, concejales municipales y miembros de juntas parroquiales rurales, se aplicó el siguiente procedimiento:

- En las circunscripciones donde se elegían dos escaños se aplicó una regla mayoritaria mediante la cual el primer puesto se asignaba a la lista que obtenía el mayor número de votos y el segundo, a la que le seguía en votos siempre que hubiese obtenido por lo menos el 35\% de los votos de la primera; caso contrario, ambos puestos correspondían a la lista más votada.

- En las circunscripciones con tres o más escaños a asignarse, se aplicó una regla proporcional, estableciéndose el método de Webster para 
la asignación de escaños, determinando que la suma de los votos alcanzados por cada una de las listas, se dividieran la serie de números 1, 3, 5, 7, etc., hasta obtener tantos cocientes como puestos por asignarse. Los cocientes obtenidos se ordenaban de mayor a menor y, se asignaban a cada lista los puestos que le correspondían de acuerdo con los más altos cocientes.

Se determinó una salvedad cuando todos los cocientes correspondieran a una sola lista, asignando el último escaño a la lista que seguía en votación. En caso de empate, se debía hacer un sorteo para definir la lista ganadora; y, los escaños alcanzados por las listas eran asignados a los candidatos según el orden en la lista.

Con relación a las circunscripciones electorales, los artículos 118 y 119 de la Constitución 2008 determinaron en 15 el número de asambleístas a ser elegidos a nivel nacional y a nivel provincial señala que sean mínimo 2 , por cada provincia, y uno más por cada doscientos mil habitantes o fracción.

De esta forma, se escogieron 124 asambleístas repartidos en 15 asambleístas nacionales en circunscripción nacional; 6 asambleístas del extranjero y 103 asambleístas provinciales, repartidos en 24 circunscripciones provinciales, de las cuales había dos grandes: Guayas con 17 escaños y Pichincha con 12, una mediana: Manabí, con 8 escaños, y 21 circunscripciones pequeñas de entre 2 y 5 escaños.

De esta manera, en las elecciones de 2009, utilizando una combinación del método de Webster con un conjunto de circunscripciones grandes, medianas y pequeñas, se logró que la proporción de los escaños adjudicados sea bastante cercana a la proporción de la votación obtenida por cada partido, como se puede ver en la Tabla 1.

\section{Tabla 1:}

Elecciones Legistativas 2009 


\begin{tabular}{|c|c|c|c|c|c|c|}
\hline \multirow{2}{*}{ Partido / Movimiento } & \multicolumn{4}{|c|}{ Asambleístas } & \multicolumn{2}{|c|}{ Porcentajes A. Prov. } \\
\hline & Nac. & Prov. & Ext. & Total & \% Votos & \% Escaños \\
\hline $\begin{array}{l}\text { Movimiento Alianza PAIS } \\
\text { (PAIS) }\end{array}$ & 7 & 47 & 5 & 59 & $42 \%$ & 45,63 \\
\hline $\begin{array}{l}\text { Partido Sociedad Patrióti- } \\
\text { ca } 21 \text { de Enero (PSP) }\end{array}$ & 3 & 16 & 0 & 19 & $15,00 \%$ & 15,53 \\
\hline $\begin{array}{l}\text { Partido Social Cristiano } \\
\text { (PSC) }\end{array}$ & 2 & 9 & 0 & 11 & $13,00 \%$ & 8,74 \\
\hline $\begin{array}{l}\text { Partido Renovador Insti- } \\
\text { tucional Acción Nacional } \\
\text { (PRIAN) }\end{array}$ & 1 & 5 & 1 & 7 & $6 \%$ & 4,85 \\
\hline Movimientos Provinciales & N/A & 7 & N/A & 7 & $5 \%$ & 5,65 \\
\hline $\begin{array}{l}\text { Movimiento Popular } \\
\text { Democrático (MPD) }\end{array}$ & 1 & 4 & 0 & 5 & $4,50 \%$ & 3,88 \\
\hline $\begin{array}{l}\text { Movimiento Municipalista } \\
\text { por la Integridad Nacional } \\
\text { (MMIN) }\end{array}$ & 0 & 5 & 0 & 5 & $4,50 \%$ & 4,85 \\
\hline $\begin{array}{l}\text { Movimiento de Unidad } \\
\text { Plurinacional Pachakutik } \\
\text { (MUPP-NP) }\end{array}$ & 0 & 4 & 0 & 4 & $4,00 \%$ & 3,88 \\
\hline $\begin{array}{l}\text { Partido Roldosista Ecua- } \\
\text { toriano (PRE) }\end{array}$ & 1 & 2 & 0 & 3 & $4,00 \%$ & 1,94 \\
\hline $\begin{array}{l}\text { Izquierda Democrática } \\
\text { (ID) }\end{array}$ & 0 & 2 & 0 & 2 & $1 \%$ & 1,94 \\
\hline $\begin{array}{l}\text { Partido Socialista-Frente } \\
\text { Amplio (PS-FA) }\end{array}$ & 0 & 1 & 0 & 1 & $0,5 \%$ & 0,97 \\
\hline $\begin{array}{l}\text { Movimiento Concertación } \\
\text { Nacional Democrática } \\
\text { (MCND) }\end{array}$ & 0 & 1 & 0 & 1 & $0,5 \%$ & 0,97 \\
\hline Total & 15 & 103 & 6 & 124 & $100 \%$ & \\
\hline
\end{tabular}

El Sistema Electoral para las Elecciones Generales de 2013

El 27 de julio de 2009, el "Congresillo" aprobó la Ley Orgánica Electoral y de Organizaciones Políticas de la República del Ecuador, Código de la Democracia (LOEOP), que derogó a la Ley Orgánica de Elecciones, a la Ley Orgánica de Control de Gasto Electoral y Propaganda Electoral, a la Ley de 
Partidos Políticos y a la Ley Orgánica para el Ejercicio del Derecho de los Ecuatorianos Domiciliados en el Exterior. Se estableció que la ley entraría a regir luego de las elecciones generales de 2009.

En la LOEOP se determinó que se seguirían eligiendo 15 asambleístas en circunscripción nacional, 2 asambleístas por las circunscripciones especiales del exterior para un total de 6 , y que seguirían eligiéndose al menos 2 asambleístas por provincia o distrito metropolitano, y uno más por cada doscientos mil habitantes o fracción que supere los 150.000, de acuerdo al último censo, pero se estableció que las circunscripciones electorales en donde se eligieran entre 8 y 12 representantes se subdividieran a su vez en 2 circunscripciones, que aquellas que pasen de 13 y hasta 18 se subdividieran en 3 y las que pasan de 18 se subdividieran en 4 circunscripciones (LOEOP, 2009, Art. 150).

De esta forma, se eliminaron del sistema electoral ecuatoriano las circunscripciones grandes, quedando divido en 30 circunscripciones pequeñas de entre 2 y 5 escaños y una circunscripción mediana de 6 escaños (La provincia de Los Ríos).

En cuanto al método de asignación de escaños, el Art. 164 de la LOEOP determinó que para asambleístas se utilizaría el método de Hare. Hare no es un método de divisores continuos como Webster o D'Hondt, sino un método o fórmula de cocientes, mediante la cual se divide la suma total de votos para el número de escaños a repartirse, obteniendo un "cociente de distribución". La asignación de escaños se hace en base al número de veces que el cociente de distribución cabe en la votación de cada partido.

Este procedimiento regularmente se complementa con el método de "restos" para asignar todos los escaños, que también fue recogido por el Art. 164 de la Ley, determinando que los escaños que faltaren por asignar corresponderán a las listas que alcanzaren las más altas aproximaciones decimales del cociente obtenido.

El método o fórmula de Hare, combinado con el método de resto mayor, como en el caso del Art. 164 de la LOEOP, tienden a favorecer a los partidos pequeños, y en general, las fórmulas de cociente electoral tienen a ser más proporcionales que las de divisores continuos (Nohlen 2004: 76-78). Este era el 
sistema electoral que debía regir para las elecciones generales que debían llevarse a cabo hasta el 20 de enero de 2013, pero el oficialismo tenía otros planes.

En diciembre de 2011, la mayoría de Alianza PAIS en la Asamblea Nacional aprobó varias reformas a la LOEOP, entre las cuales estaba la modificación del método de asignación de escaños de Hare a Webster. Remitido al Ejecutivo para su aprobación, el presidente Correa vetó el proyecto y cambió el método de asignación de escaños para asambleístas provinciales por el método D'Hondt. En cuanto a los nacionales, se aceptó el método de Webster. La mayoría oficialista de la Asamblea se allanó totalmente al veto presidencial y la modificación a la LOEOP entró en vigor el 6 de febrero de 2012.

Por mandato constitucional, las reformas legales aprobadas en febrero de 2011 no podían entrar a regir hasta después de las elecciones que debían llevarse a cabo en enero de 2013, puesto que el Art. 117 de la carta política prohíbe la realización de reformas legales en materia electoral durante el año anterior a la celebración de elecciones.

Ante esto, el CNE nombrado en 2011, afín al oficialismo, amparado en sus supuestas competencias, decidió mover el calendario electoral al 19 de febrero de 2013 a fin de que las reformas implementadas el 6 de febrero de 2012 pudieran tener efecto en el sistema electoral que las regularía. Con esta modificación del método de asignación de escaños, Alianza PAIS, con una votación del 52.30\%, logró obtener 91 escaños de 116 de asambleístas provinciales, es decir que con poco más de la mitad de los votos, obtuvo más del casi el 75\% de los escaños (Tabla 2).

\section{Tabla 2:}

Elecciones Legislativas 2013

\begin{tabular}{|l|c|c|c|c|c|c|}
\hline \multirow{2}{*}{ Partido / Movimiento } & \multicolumn{3}{|l|}{ Asambleístas } & \multicolumn{2}{l|}{ Porcentajes A. Prov } \\
\cline { 2 - 7 } & Nac. & Prov. & Ext. & Total & $\%$ Votos & $\%$ Escaños \\
\hline $\begin{array}{l}\text { Movimiento Alianza } \\
\text { PAIS (PAIS) }\end{array}$ & 8 & 86 & 6 & 100 & $52,30 \%$ & 74,14 \\
\hline $\begin{array}{l}\text { Movimiento Creando } \\
\text { Oportunidades (CREO) }\end{array}$ & 2 & 8 & 0 & 10 & $11,42 \%$ & 6,90 \\
\hline
\end{tabular}




\begin{tabular}{|l|c|c|c|c|c|c|}
\hline $\begin{array}{l}\text { Partido Social Cristiano } \\
\text { (PSC) }\end{array}$ & 1 & 6 & 0 & 7 & $8,99 \%$ & 4,38 \\
\hline $\begin{array}{l}\text { Partido Sociedad Patrió- } \\
\text { tica 21 de Enero (PSP) }\end{array}$ & 1 & 4 & 0 & 5 & $5,64 \%$ & 2,92 \\
\hline $\begin{array}{l}\text { Partido Avanza (AVAN- } \\
\text { ZA) }\end{array}$ & 0 & 5 & 0 & 5 & $2,92 \%$ & 3,65 \\
\hline $\begin{array}{l}\text { Movimiento de Unidad } \\
\text { Plurinacional Pachakutik } \\
\text { (MUPP) }\end{array}$ & 1 & 4 & 0 & 5 & $4,72 \%$ & 3,65 \\
\hline $\begin{array}{l}\text { Movimientos Provin- } \\
\text { ciales }\end{array}$ & N/A & 3 & N/A & 3 & $6,28 \%$ & 2,19 \\
\hline $\begin{array}{l}\text { Partido Roldosista Ecua- } \\
\text { toriano (PRE) }\end{array}$ & 1 & 0 & 0 & 1 & $4,51 \%$ & 0,00 \\
\hline $\begin{array}{l}\text { Movimiento Sociedad } \\
\text { Unida Más Acción } \\
\text { (SUMA) }\end{array}$ & 1 & 0 & 0 & 1 & $3,22 \%$ & 0,00 \\
\hline Total & 15 & 116 & 6 & 137 & $100,00 \%$ & \\
\hline
\end{tabular}

En las Tabla 1 y 2 se puede ver incluso que, mientras en 2009 existían organizaciones políticas que obtuvieron al menos un escaño con votaciones inferiores al 1\%, en 2013 organizaciones políticas con porcentajes de entre el $3 \%$ y el $5 \%$ no obtuvieron ningún escaño ${ }^{12}$.

Pese a lo marcadamente desproporcional que es el sistema electoral puesto en vigencia en 2013, se conservaron las mismas reglas para las elecciones generales de 2017, manteniéndose la desproporción entre la votación y la asignación de escaños, como puede verse en la Tabla 3. Elecciones en las que nuevamente el partido oficialista se hizo con la mayor parte de los escaños en disputa, logrando obtener nuevamente mayoría absoluta, aunque, esta vez, no logró la mayoría calificada de los dos tercios de la Asamblea, que sí tenía en la conformación anterior ${ }^{13}$.

12.- "Por tanto, en un ejercicio ilustrativo, al domino de las circunscripciones pequeñas se le puede atribuir un $50 \%$ del efecto concentrador del sistema, al método D'Hondt aproximadamente un $30 \%$ y el $20 \%$ a otros elementos fuera del sistema electoral. Hay que considerar que todo sistema electoral tiene un efecto reductor en el número de partidos que participan en la contienda electoral; sin embargo, para que un sistema sea calificado como proporcional no debería permitir grados de desproporcionalidad mayores a los diez IG." (Ortiz, 2016: 24)

13.- La pérdida de escaños de Alianza País en la Asamblea legislativa de 2017 pasa más por su votación, que fue más de 10 puntos porcentuales menor a la obtenida en las elecciones de 2013, lo que puede deberse a una pérdida de popularidad del gobierno por la crisis económica, la no participación de Rafael Correa en las elecciones y la menor cantidad de organizaciones políticas disputándose los escaños en juego, que al sistema electoral, que sigue favoreciendo a los partidos mayoritarios. 


\section{Tabla 3:}

Elecciones Legislativas 2017

\begin{tabular}{|l|c|c|c|c|c|c|}
\hline \multirow{2}{*}{\multicolumn{1}{|c|}{ Partido / Movimiento }} & \multicolumn{5}{c|}{ Asambleístas } & \multicolumn{2}{c|}{ Porcentajes A. Prov } \\
\cline { 2 - 8 } & Nac. & Prov. & Ext. & Total & \% Votos & \% Escaños \\
\hline $\begin{array}{l}\text { Movimiento Alianza PAIS } \\
\text { (PAIS) }\end{array}$ & 7 & 67 & 4 & 74 & 39,07 & 48,91 \\
\hline Alianza CREO-SUMA & 3 & 31 & 2 & 34 & 21,65 & 22,63 \\
\hline Partido Social Cristiano (PSC) & 3 & 12 & 0 & 15 & 15,09 & 8,76 \\
\hline $\begin{array}{l}\text { Movimiento de Unidad Plurina- } \\
\text { cional Pachakutik (MUPP) }\end{array}$ & 0 & 4 & 0 & 4 & 4,67 & 2,92 \\
\hline Izquierda Democrática (ID) & 1 & 3 & 0 & 4 & 4,77 & 2,19 \\
\hline Movimientos Provinciales & N/A & 3 & N/A & 3 & 6,06 & 2,19 \\
\hline $\begin{array}{l}\text { Partido Sociedad Patriótica 21 } \\
\text { de Enero (PSP) }\end{array}$ & 0 & 2 & 0 & 2 & 3,94 & 1,46 \\
\hline Partido Fuerza Ecuador (FE) & 1 & 0 & 0 & 1 & 4,75 & 0,00 \\
\hline Total & 15 & 116 & 6 & 137 & 100 & \\
\hline
\end{tabular}

A fin de tratar de graficar de mejor manera la grave desproporcionalidad generada por el sistema electoral actual, se ha realizado un ejercicio comparativo con la votación de la provincia del Guayas, la circunscripción electoral más grande hasta antes de la promulgación de la LOEOP, que la dividió en 4 circunscripciones pequeñas. Se ha tomado para el ejercicio únicamente las elecciones de 2013 porque el porcentaje de votos de Alianza PAIS es mayor y permite ver de mejor forma la desproporcionalidad del sistema.

En la Tabla 4, se puede observar los resultados reales de las elecciones de 2013, en dónde se aprecia cómo se repartieron los escaños únicamente entre los dos partidos más grandes, existiendo una desproporcionalidad en la asignación de 25 puntos porcentuales. Es decir, en los términos descritos por Nohlen y Ortiz previamente, el sistema ha dejado de ser proporcional para transformarse en mayoritario, a pesar de la fórmula proporcional. 


\section{Tabla 4:}

Resultados Guayas 2014 (D' Hondt, 4 distritos)

\begin{tabular}{|l|c|c|c|c|c|c|c|c|}
\hline & & & \multicolumn{6}{|c|}{ ESCAÑOS } \\
\hline PARTIDOS & Votos & \% Votos & D1 & D2 & D3 & D4 & TOTAL & \% Escaños \\
\hline PAIS & 4232852 & $55 \%$ & 4 & 4 & 3 & 5 & 16 & 80 \\
\hline PSC - MDG & 1697548 & $22 \%$ & 1 & 1 & 2 & 0 & 4 & 20 \\
\hline PRIAN & 451553 & $6 \%$ & 0 & 0 & 0 & 0 & 0 & - \\
\hline PRE & 420451 & $5 \%$ & 0 & 0 & 0 & 0 & 0 & - \\
\hline $\begin{array}{l}\text { A.U.P. D.IZ- } \\
\text { QUIERDAS }\end{array}$ & 177949 & $2 \%$ & 0 & 0 & 0 & 0 & 0 & - \\
\hline PSP & 264092 & $3 \%$ & 0 & 0 & 0 & 0 & 0 & - \\
\hline SUMA & 201981 & $3 \%$ & 0 & 0 & 0 & 0 & 0 & - \\
\hline AVANZA & 178564 & $2 \%$ & 0 & 0 & 0 & 0 & 0 & - \\
\hline RUPTURA & 87311 & $1 \%$ & 0 & 0 & 0 & 0 & 0 & - \\
\hline P.S. - F. A. & 58253 & $1 \%$ & 0 & 0 & 0 & 0 & 0 & - \\
\hline TOTAL & 7770554 & $100,00 \%$ & 5 & 5 & 5 & 5 & 20 & 100 \\
\hline
\end{tabular}

En la Tabla 5 se ha realizado una simulación que mantiene el método D'Hondt, pero cambia la circunscripción a una sola de 20 escaños, es decir una circunscripción grande. Como se puede observar, la desproporcionalidad baja sustancialmente, aunque en el caso del partido mayoritario aún mantiene una diferencia de 10 puntos porcentuales, mientras que dos partidos minoritarios se hacen con un escaño cada uno.

\section{Tabla 5:}

Simulación Resultados Guayas 2014 (D' Hondt, 1 distritos)

\begin{tabular}{|l|c|c|c|c|}
\hline \multicolumn{1}{|c|}{ PARTIDOS } & Votos & \% Votos & Escaños & \% Escaños \\
\hline PAIS & 4232852 & $55 \%$ & 13 & 65 \\
\hline PSC - MDG & 1697548 & $22 \%$ & 5 & 25 \\
\hline PRIAN & 451553 & $6 \%$ & 1 & 5 \\
\hline PRE & 420451 & $5 \%$ & 1 & 5 \\
\hline
\end{tabular}




\begin{tabular}{|l|c|c|c|c|}
\hline A.U.P. D.IZQUIERDAS & 177949 & $2 \%$ & 0 & - \\
\hline PSP & 264092 & $3 \%$ & 0 & - \\
\hline SUMA & 201981 & $3 \%$ & 0 & - \\
\hline AVANZA & 178564 & $2 \%$ & 0 & - \\
\hline RUPTURA & 87311 & $1 \%$ & 0 & - \\
\hline P.S. - F. A. & 58253 & $1 \%$ & 0 & - \\
\hline TOTAL & 7770554 & $100,00 \%$ & 20 & \\
\hline
\end{tabular}

Finalmente, en la Tabla 6 se realiza una simulación de resultados, utilizando el método de Hare, anterior a la reforma de la LOEOP de 2012 y que nunca se puso en práctica. Como se puede ver, la asignación de escaños deja de ser desproporcional y más partidos minoritarios se hacen adjudicatarios de escaños.

Tabla 6:

Simulación Resultados Guayas 2014 (Hare)

\begin{tabular}{|l|c|c|c|c|c|c|}
\hline \multicolumn{1}{|c|}{ PARTIDOS } & Votos & \% Votos & $\begin{array}{c}\text { Escaños } \\
\text { (sin resto) }\end{array}$ & Resto & $\begin{array}{c}\text { Escaños } \\
\text { finales }\end{array}$ & $\begin{array}{c}\text { \% Esca- } \\
\text { nos }\end{array}$ \\
\hline PAIS & 4.232 .852 & $55 \%$ & 11 & 333429 & 12 & 60 \\
\hline PSC - MDG & 1.697 .548 & $22 \%$ & 4 & 279576 & 5 & 25 \\
\hline PRIAN & 451.553 & $6 \%$ & 1 & 97060 & 1 & 5 \\
\hline PRE & 420.451 & $5 \%$ & 1 & 65958 & 1 & 5 \\
\hline $\begin{array}{l}\text { A.U.P. D.IZ- } \\
\text { QUIERDAS }\end{array}$ & 177.949 & $2 \%$ & 0 & 177949 & & 0 \\
\hline PSP & 264.092 & $3 \%$ & 0 & 264092 & 1 & 5 \\
\hline SUMA & 201.981 & $3 \%$ & 0 & 201981 & & 0 \\
\hline AVANZA & 178.564 & $2 \%$ & 0 & 178564 & & 0 \\
\hline RUPTURA & 87.311 & $1 \%$ & 0 & 87311 & & 0 \\
\hline P.S. - F. A. & 58.253 & $1 \%$ & 0 & 58253 & & 0 \\
\hline TOTAL & 7.770 .554 & $100 \%$ & 17 & & 20 & \\
\hline
\end{tabular}




\section{Conclusiones}

En este trabajo se ha podido ver como el gobierno de Alianza PAIS y Rafael Correa, a través de la mayoría parlamentaria inicial que consiguió en 2008 para la Asamblea Constituyente, logró hacerse con el control de dos instituciones fundamentales para el manejo del sistema electoral: el CPCCS y el CNE. La mayoría oficialista que llegó a la Asamblea Constituyente eligió vocales afines al régimen para integrar el CNE de transición, quienes, a su vez, nombraron también miembros cercanos al régimen para el CPCCS, los que, por supuesto, nombraron consejeros afines al oficialismo para integrar el CNE definitivo.

El control obtenido por el oficialismo de la Función de Transparencia y Control Social y de la Función Electoral le permitió, a su vez, manipular el sistema electoral, aumentando su tendencia concentradora a fin de favorecer a su partido y maximizar sus resultados electorales, en desmedro de los partidos de oposición, los que, cabe reconocer, han sido incapaces de articular alianzas que les permitieran participar de forma menos fragmentada en las elecciones, lo que hubiera incidido en el número de escaños que podrían haber obtenido (Ortiz 2016: 27).

Esta manipulación del sistema electoral se logró distorsionando el principio democrático de igualdad por medio de dos elementos fundamentales del sistema electoral: la circunscripción electoral y el método de adjudicación de escaños, combinando una fórmula de por sí menos proporcional que otras, la de D'Hondt, con circunscripciones pequeñas en las que hay menos escaños a repartir, lo que además constituye una violación de la Constitución.

Como señala Nohlen, las circunscripciones pequeñas favorecen fuertemente a los partidos grandes, como en este caso a Alianza PAIS, presentando diferencias significativas en el nivel nacional entre la proporción de votos y escaños, perjudicando a los partidos pequeños.

En sintesis, la aplicación de la proporcionalidad como fórmula de decisión en circunscripciones pequeñas no conduce al objetivo de representación proporcional sino al de representación por mayoría. El sistema proporcional en circunscripciones electorales pequeñas actúa, de hecho, como un sistema 
mayoritario (Nohlen 1995: 48).

Asimismo, como señalan Basabe y Martínez, las reformas electorales permitieron a Alianza PAIS controlar más de las dos terceras partes de la Asamblea Nacional y a través de ello, viabilizar la agenda política del Ejecutivo. Inclusive, la mayoría absoluta de AP facilitó una reforma constitucional que dio paso a la reelección indefinida, otra característica de los modelos autoritario-competitivos (2014:159).

Las elecciones en Ecuador durante la década el correísmo fueron libres, sin embargo, el análisis presentado en este trabajo demuestra que no fueron justas. La cancha estuvo inclinada a favor del partido oficialista a través de las reformas electorales facilitadas por un Consejo Nacional Electoral afín al ejecutivo.

Así, este trabajo muestra que el gobierno de Alianza PAIS, a través del control en la elección de las autoridades electorales, desniveló el campo de juego en desmedro de la oposición, lo que viola flagrantemente uno de los atributos esenciales de una democracia; esto es, la existencia de elecciones justas y libres, configurando, sólo por ese hecho, un autoritarismo competitivo en el Ecuador, de acuerdo a lo señalado por Levitsky y Way (2010: 366).

Finalmente, existe también abundante evidencia empírica de que durante el gobierno de Rafael Correa se dió un trato desigual a la oposición, tanto en el acceso a los medios, como en el financiamiento en sí de las campañas, con un gobierno haciendo campaña sin control alguno en los medios públicos e inaugurando obras justamente en época de campaña. Este trato desigual también representa una cancha inclinada, sin embargo, no constituye parte del objeto de estudio de este trabajo ${ }^{14}$.

14.- En julio de 2019 la Fiscalia General del Estado abrió una investigación sobre presuntos aportes entregados por varias empresas multinacionales al movimiento Alianza PAIS desde noviembre de 2013 a febrero de 2014, periodo en el que el expresidente Rafael Correa lideraba esa organización política. Según la denunciante, la fundación Mil Hojas, las donaciones alcanzarían los 11,6 millones de dólares. 


\section{Biografía}

Basabe, Santiago y Julián Martínez (2014). "Ecuador: Cada ez menos democracia, cada vez más autoritarismo....con elecciones". Revista de Ciencia Política Vol. 34.

Consejo Nacional Electoral www.cne.gob.ec

Constitución de la Republica, (2008), Registro Oficial 490

Diamond, Larry (2009). "Thinking about Hybrid Regimes". En Diamond, Larry y Marc F. Plattner (Eds.) Democracy: A Reader. Baltimore: The Johns Hopkins University Press and the National Endowment for Democracy

Diamond, Larry (2015). "Facing Up to the Democratic Recession," Journal of Democracy 26, no. 1 (January 2015), páginas 141-55.

El Comercio (2010). "La política marcó la hoja de ruta del Consejo", 14 de marzo

El Universo (2012). “José Domingo Paredes, la Pieza Clave de lo Electoral”, 12 de agosto.

El Universo (2017). "Coincidencias con AP marcan presencia de Pozo en el CNE", 1 de mayo.

Estrella Aguilar, Rafael (2018). Webster vs. D'Hondt. Cuenca, Universidad de Cuenca.

Expreso (2011). "Domingo Paredes dirigirá Consejo Electoral", 29 de noviembre.

Foa, Roberto Stefan y Yascha Mounk (2016). "The Democratic Disconnect" Journal of Democracy 27, no. 3, julio, páginas 5-17. 
Freidenberg, Flavia (2011). El Código de la Democracia: una evaluación del proceso de reforma electoral del Ecuador (2008-2009). Ágora Democrática,

Gallagher, Michael (1991) "Proportionality, disproportionality and electoral systems", Electoral Studies, Volume 10, Issue 1, marzo, páginas 33-51

Levistky, Steven y Daniel Ziblatt (2018). How Democracies Die. Nueva York: Crown Publishing

Levitsky, Steven y Lucan Way (2009). "The Rise of Competitive Authoritarianism". En Diamond Larry y Marc F. Plattner Democracy: A Reader. Baltimore: The Johns Hopkins University Press and the National Endowment for Democracy

Levitsky, Steven y Lucan Way (2010). Competitive Authoritarianism. Hybrid Regimes After the Cold War. Cambridge: Cambridge University Press

Ley Orgánica Electoral y de Organizaciones Políticas, LOEOP (2009). Registro Oficial Suplemento No. 578 de 27 de abril de 2009

Lührmann, Anna y Staffan I. Lindberg (2019). “A third wave of autocratization is here: what is new about it?". Journal Democratization, Volume 26, 2019 - Issue 7, páginas 1095-1113

Mainwaring, Scott, Daniel Brinks y Aníbal Pérez-Liñán (2007). “Classifying political regimes in Latin America, 1945-2004”. En Munck Gerardo L. (Ed.). Regimes and democracy in Latin America: theories and methods. Oxford: Oxford University Press

Moscoso, Arturo. (2013). Autoritarismo y neoliberalismo en Latinoamérica: ¿están relacionados?. Recuperado de https://repositorio.flacsoandes. edu.ec/bitstream/10469/6144/2/TFLACSO-2013ARMM.pdf 
Nohlen, Dieter (1995). Elecciones y sistemas electorales. Caracas: Editorial NUEVA SOCIEDAD.

Nohlen, Dieter (2004). Sistemas electorales y partidos políticos. México: Fondo de Cultura Económica.

O’Donnell, Guillermo (1997). Contrapuntos: ensayos escogidos sobre autoritarismo y democratización. Buenos Aires: Paidós

Ortiz Ortiz, Richard (2016). "Las singularidades del sistema electoral legislativo en Ecuador". En Ortiz Ortiz, Richard (Ed.), Reforma electoral en Ecuador. Estudios sobre el Sistema electoral para la Asamblea Nacional y sobre el voto electrónico. Quito: Universidad de las Américas.

Pachano, Simón y Sergio García (2015). "Ecuador: un régimen híbrido". Revista Latinoamericana de Política Comparada 10: 123-148.

Sanchez-Sibony, Omar (2017). "Classifying Ecuador's Regime under Correa: A Procedural Approach". Journal of Politics in Latin America, 9, 3, páginas 121-140.

Schedler, Andreas (2006). "The Logic of Electoral Authoritarianism". En Schedler, Andreas (Ed.), Electoral Authoritarianism. The Dynamics of Unfree Competion. Boulder: Lynne Rienner Publishers

Zakaria, Fareed, (1998). El surgimiento de la democracia iliberal. Quito: Programa de Apoyo al Sistema de Gobernabilidad Democrática. 